\title{
Characterization of the Organic LED Based Visible Light Communications
}

Petr Chvojka ${ }^{1}$, Petr Dvorak ${ }^{1}$, Petr Pesek ${ }^{1}$, Stanislav Zvanovec ${ }^{1}$, Paul Anthony Haigh ${ }^{2}$, Zabih Ghassemlooy $^{3}$ ${ }^{\prime}$ Department of Electromagnetic Field, Faculty of Electrical Engineering, Czech Technical University in Prague, Czech Republic ${ }^{2}$ High Performance Networks Group, Faculty of Engineering, University of Bristol, BS8 1TH, UK

${ }^{3}$ Optical Communications Research Group, NCRLab, Faculty of Engineering and Environment, Northumbria University, Newcastle upon Tyne, NE1 8ST, UK

Corresponding authors: chvojpe8@fel.cvut.cz,xzvanove@fel.cvut.cz

\begin{abstract}
This paper presents experimental results on a visible light communications system utilizing organic light-emitting diodes (OLEDs) and 16-quadrature amplitude modulation. The modulation bandwidth of the OLED device is limited The modulation bandwidh of the OLED device is limited to $310 \mathrm{kHz}$ and the signal is generated on carrier frequencies $f_{c}=\{100,200,300\} \mathrm{kHz}$ for use across different regions of the OLED frequency response. We demonstrate how setting a specific carrier frequency can affect the system performance. Th supported bit rates are 220,640 and $260 \mathrm{~kb} / \mathrm{s}$ for $f_{c}=100,200$ and $300 \mathrm{kHz}$, respectively. The angular dependence of the system performance is also analysed.
\end{abstract}

Keywords-bit error rate; organic light emitting diode; visible light communications

\section{INTRODUCTION}

Organic light emitting diodes (OLEDs) have attracted both researchers and industrialists for their potential for employment in 'green' energy efficient applications such as solid-state lighting (SSL) or high-resolution display technologies. Moreover, their properties enable utilization in visible light communication (VLC) systems [1]. In spite of the fact that traditional LEDs made from inorganic materials are the most popular optical sources due to a high optical output power and substantially wider modulation bandwidths (several $\mathrm{MHz}$ [2]), OLEDs represent an exciting subject for research. They offer very low production costs across their large area panels, which is in complete contrast with traditional LEDs that usually serve as a point source. On the other hand, their $3 \mathrm{~dB}$ modulation bandwidth is limited within the $\mathrm{kHz}$ region $[3,4]$.

Organic semiconductors can be solution processed, which reduces the cost significantly. Typical OLEDs consist of thin film organic materials placed between two oppositely polarized electrodes, where one is typically indium tin oxide (ITO) due to being transparent for light emission. The typical luminous efficiency of OLEDs is $40-60 \mathrm{~lm} / \mathrm{W}$ [5], which represents a hug disadvantage in comparison to typical inorganic efficiencies of $120 \mathrm{~lm} / \mathrm{W}$, with the values up to $200 \mathrm{~lm} / \mathrm{W}$ already demonstrated [6]. This is not expected to be a long term challenge, however, as white OLEDs have been reported with values of $150 \mathrm{~lm} / \mathrm{W}$ [7], making them suitable for future SSL and VLC systems.

Despite OLEDs still being in their infancy, one major of their major applications to date is slow speed messaging and data transfers within the IEEE $802.15 .7 \mathrm{r}$ standard $[8,9]$, where significant results have been demonstrated in organic VLC (OVLC). For example, $10 \mathrm{Mb} / \mathrm{s}$ and $20 \mathrm{Mb} / \mathrm{s}$ transmission capacities were experimentally achieved using an OLED with $270 \mathrm{kHz}$ [10] and $350 \mathrm{kHz}$ [11] bandwidth, respectively. Both experiments were based on custom designed organic polymer LEDs (PLEDs) and equalization techniques such as least mean square (LMS) and multilayer perceptron (MLP) artificial neural network (ANN) filtering [5] were employed. The highest data rate ever achieved in OVLC was reported in [12], where wavelength division multiplexing (WDM) and an MLP-ANN wavelength division multiplexing (WDM) and an MLP-ANN equalizer were adopted for individual PLED chips. The red and
blue components supported $27.9 \mathrm{Mb} / \mathrm{s}$ and $18.6 \mathrm{Mb} / \mathrm{s}$, respectively, while the green component offered $8.4 \mathrm{Mb} / \mathrm{s}$. Thus, an aggregated capacity of $\sim 55 \mathrm{Mb} / \mathrm{s}$, and an $\sim 2.75$-fold increase over the previously reported highest data rate [11]. This also represents an $\sim 100$ times gain over the first reported OVLC system [5], which is a remarkable improvement over the last 4 years.

The most popular modulation scheme employed in VLC is on-off keying (OOK) [2], however, recently spectral efficiency modulation formats such as orthogonal division multiplexing (13,14] and multiband carrier-less amplitude and phase ( $m$-CAP) modulation have been adopted $[15,16]$. Thus, in this paper we present a test of the commercially available OLED panel LG N6SA30 using quadrature amplitude modulation (QAM), which was utilized in the aforementioned OFDM and $m$-CAP VLC systems. We focus explicitly on the OLED performance, and therefore did not enploy an equalizer, which can further boost the transmission rate We show that transmitting the signal with $f_{c}=200 \mathrm{kHz}$ within the OLED bandwidth can provide data rates up to $640 \mathrm{~kb} / \mathrm{s}$ within the $7 \%$ FEC limit. Moreover, angular dependency shows that the OVLC system support data rates of $160 \mathrm{~kb} / \mathrm{s}$ with a tilt angle up to $48^{\circ}$. This is the important result because it could lead to use in advanced techniques, e.g., angle diversity.

The rest of the paper is organized as follows: OLED characteristics and experimental setup description are given in Section II. The results are illustrated in Section III and conclusions are drawn in Section IV. 
II. EXPERIMENTAl MEASUREMENT

A. OLED characteristics

All electro-optic devices can be characterized by their $L-I-V$ transfer function, where $L$ is optical power, $I$ is drive current and $V$ is forward voltage. The measured L-I-V curve of the OLED LG N6SA30 is illustrated in Fig. 1. To ensure that the OLED is operating in the linear region, we set the bias current to $\sim 150 \mathrm{~mA}$. The OLED radiation spectrum was measured using a Yokogawa AQ6370C spectrometer, see inse in Fig. 1, with four major peaks emerging at X, Y, Z, M nm.

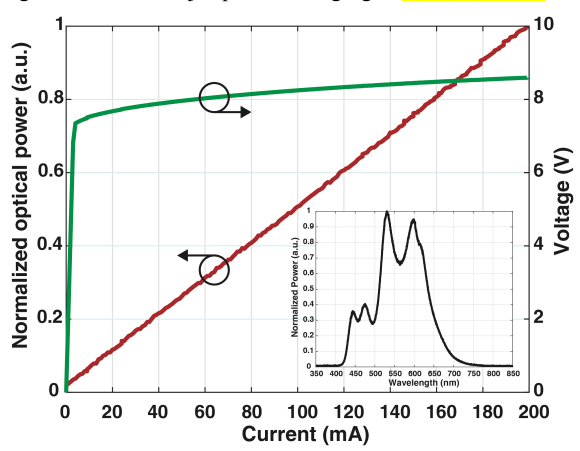

Fig. 1. Measured L-I-V curve of the tested OLED (inset shows measured radiation spectrum)

As mentioned, the ability to produce large area panels gives OLEDs an advantage over traditional inorganic diodes. Nevertheless, it also reduces the $3 \mathrm{~dB}$ modulation bandwidth, since organic devices have a capacitor-like behaviour exhibiting low-pass filter character. The plate capacitance is given by:

$$
C=\frac{A \varepsilon_{0} \varepsilon_{r}}{d}
$$

where $A$ and $d$ are OLED photoactive area and thickness, respectively, and $\varepsilon_{0}$ and $\varepsilon_{r}$ are the permittivity of vacuum and the relative dielectric constant of the organic layer. For instance, $\varepsilon_{r}$ of the organic materials such as $\mathrm{LiF}$ and $\mathrm{NaCl}$ is 9.036 and 5.895 , respectively [17]. The thickness is typically around 1-200 nm. Clearly, an increase of the active panel area results in high capacitance and consequently device bandwidth reduction. The cut-off frequency is subsequently given by:

$$
f_{c}=(2 \pi R C)^{-1}
$$

where $R$ is the series resistance of the OLED. The OLED used in this paper has $99 \mathrm{~mm} \times 99 \mathrm{~mm}$ (width $\times$ height) dimensions. Thus, the total OLED area is $9,800 \mathrm{~mm}^{2}$ inducing high device capacity and low modulation bandwidth according to (1) and (2), respectively.
The measured OLED frequency response and noise levels are illustrated in Fig. 2 showing the $3 \mathrm{~dB}$ modulation bandwidth at $310 \mathrm{kHz}$. The frequency response can be divided into 3 regions: $\mathrm{A}, \mathrm{B}$ and $\mathrm{C}$. The significant attenuation in section $\mathrm{A}$ is caused by the high cut-on frequency of the bias tee, which results in substantial attenuation of the DC and low frequency components. This is well-known as the baseline wander (BLW) phenomenon. Such a bandpass shape causes a limitation on the system transmission capacity. Several methods were introduced to overcome the BLW effect such as a high impedance NAND driving circuit [1]. Next, section $B$ shows the bandpass region of the OLED and finally, the attenuation outside the $3 \mathrm{~dB}$ bandwidth due to the low pass characteristics of the OLED is depicted in the section $\mathrm{C}$. Thus, the bandpass bandwidth is just slightly over $200 \mathrm{kHz}$ and significant attenuation outside this bandwidth should be expected.

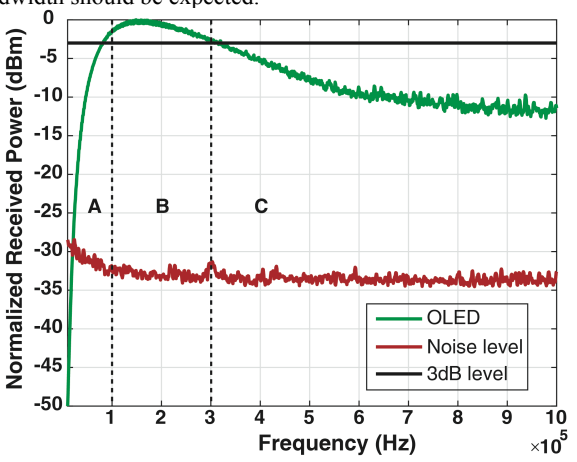

Fig. 2. Measured frequency response of the OLED (green line) and noise level (red line). The $3 \mathrm{~dB}$ bandwidth is $310 \mathrm{kHz}$. The spectrum analyser resolution and video bandwidth resolution was $5 \mathrm{kHz}$.

\section{B. Experimental setup}

The experimental test setup is depicted in Fig. 3, with photograph inset. The Rhode Schwarz SMW200A vector signal generator was used to generate predefined pseudorandom binary sequence (PRBS) of length $2^{15}-1$. The data was mapped into 16 QAM constellation symbols following Gray coding. Individual symbols were pulse shaped using a square root raised cosine (RRC) filter with a roll-off factor $\alpha=0.15$. The output signal from the signal generator was then mixed with a DC current using a bias tee ZX85-12G-S + to set the OLED operating point into the linear region. Power of the injected RF signal was $4 \mathrm{dBm}$ for all carrier frequencies. The optical signal was received by the Thorlabs detector PDA10A $(150 \mathrm{MHz}$ bandwidth) and captured by the Rhode Schwarz FSW26 spectrum analyser and further proceeded offline in MATLAB. The transmission span was set at $0.10 \mathrm{~m}$ for every measurement.

The error vector magnitude (EVM) was measured from the received constellation diagrams and the bit error rate (BER) of the system was consequently calculated by [18]: 


$$
B E R=\frac{2\left(1-\frac{1}{L}\right)}{\log _{2} L} Q\left(\sqrt{\left(\frac{3 \log _{2} L}{L^{2}-1}\right) \frac{2}{E V M_{R M S}^{2} \log _{2} M}}\right)
$$

where $L$ and $M$ are the number of levels in each dimension of the modulation system and the modulation order (here $L=4$ and $M=16$ ), respectively, and $Q(\cdot)$ is the $Q$ function.

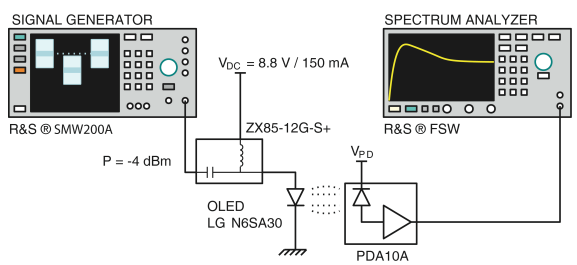

(a)
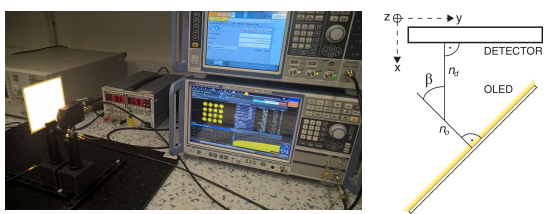

(b)

(c)

Fig. 3. (a) The experimental test setup, (b) photograph from the measurements and (c) the tilt angle definition.

The angular dependency was measured using the same setup as mentioned above for the range of $\beta=0-90^{\circ}$, where $\beta$ is the angle between the normal vectors $n_{d}$ and $n_{0}$ that are perpendicular to the receiver and OLED plane, respectively, as illustrated in Fig. 3(c)

III. RESULTS

In this section, the BER performance of the system for a range of $f_{c}$ and EVM angular dependency are analysed. We focused on setting the $f_{c}$ values correctly in order to cover different parts of the OLED frequency response. Thus, the signal is transmitted to utilize the frequencies below the cut-on and cut-off frequencies at $f_{c}=100 \mathrm{kHz}$ an $300 \mathrm{kHz}$, respectively, and using the bandpass region at $f_{c}=200 \mathrm{kHz}$, which is ideal for in-band transmission.

The BER performance for a range of symbol rates $R_{s}$ is illustrated in Fig. 4. We can see that $f_{c}=300 \mathrm{kHz}$ exhibits the worst performance for low values of $R_{s}$, while increasing $R_{s}$ towards higher values results in faster degradation of the signal for $f_{c}=100 \mathrm{kHz}$, with a crossing point at $\sim 50 \mathrm{ksym} / \mathrm{s}$. This phenomenon is caused by the increase of the total signal bandwidth being $(1+\alpha)$ times the symbol rate [19]. Clearly, the signal undergoes higher attenuation below the cut-on frequency than outside the $3 \mathrm{~dB}$ bandwidth as can be inferred from Fig. 2 . Thus, we experience higher BER performance deterioration fo the boundary values of $f_{c}$, resulting in the highest achieved bit rates $R_{b}$ of $\sim 220 \mathrm{~kb} / \mathrm{s}$ and $\sim 260 \mathrm{~kb} / \mathrm{s}$ for $f_{c}=100 \mathrm{kHz}$ and 300
$\mathrm{kHz}$, respectively, at the 7\% FEC limit. The best performance is obtained for the signal at $f_{c}=200 \mathrm{kHz}$ due to the optimal bandpass bandwidth utilization. Such system supports data rate up to $\sim 640 \mathrm{~kb} / \mathrm{s}$ at the $7 \% \mathrm{FEC}$ limit. The performance is further illustrated on the received constellation diagrams, which are shown in Fig. 4(b), for $R_{s}=80 \mathrm{ksym} / \mathrm{s}$ at $f_{c}=100 \mathrm{kHz}, 200 \mathrm{kHz}$ and $300 \mathrm{kHz}$ in their respective colours.

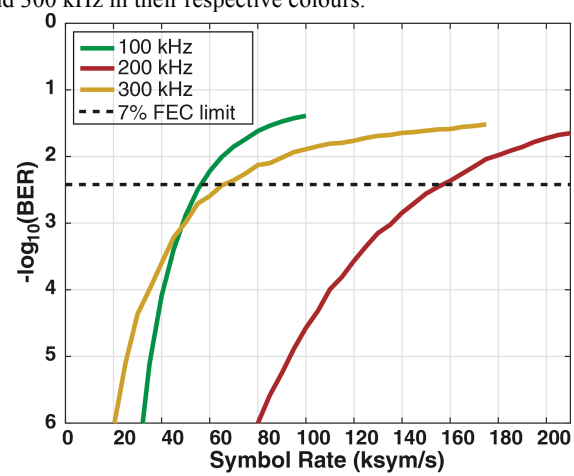

(a)
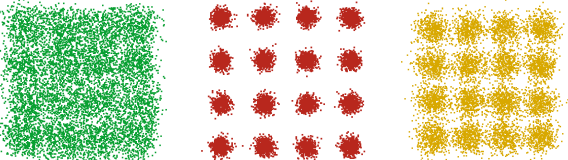

(b)

Fig. 4. (a) The measured BER for the range of $f_{c}$. The signal transferred at $c_{c}=20 \mathrm{kz}$ shows the best $\mathrm{pe}$. up to $640 \mathrm{~kb} / \mathrm{s}$ at $7 \%$ FEC limit. Fig. 4 (b) illustrates corresponding constellation diagrams for the range of $f_{c}$ in their respective colours for
$R_{s}=80 \mathrm{ksym} / \mathrm{s}$.

A very interesting feature of within the OVLC domain introduces angular dependency of the received signal from a large area panel. Both angular dependencies of the measured EVM and calculated BER performance are illustrated in Fig. 5(a) for a 16-QAM signal transmitted at $R_{s}=40 \mathrm{ksym} / \mathrm{s}$. The tilt angle $\beta$ was varied in the range $0-90^{\circ}$. Clearly, the system supports data communication with a BER $<10^{-6}$ (i.e., EVM $<\sim-20 \mathrm{~dB}$ ) main direction). Even higher tilt angles support (i.e, up to $48^{\circ}$ ) the data transmission within the $7 \%$ FEC limit (i.e., EVM $<\sim 6-15 \mathrm{~dB}$ ), which is significant for a range of applications such as mobility and protection against signal path blocking $[20,21]$. Moreover, the performance of the system is further illustrated in Fig. 5(b). The received constellation diagrams for 16-QAM, 32-QAM and 64-QAM signals at $\beta=$ $40^{\circ}$ show the subsequently decreasing performance with higher order $M$-QAM signals. 


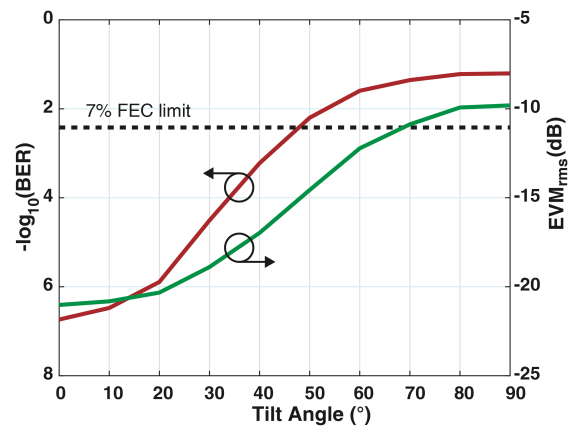

(a)

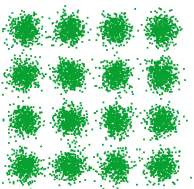

Fig. 5. (a) The BER performance and EVM angular dependency for 16-QAM signal and (b) the received constellation diagrams for $\{16,32,64\}$-QAM at $\beta=40^{\circ}$.

\section{CONCLUSIONS}

In this paper, we tested a large area OLED panel for low speed communication purposes within IEEE 802.15.7r We showed that by optimal selection of the carrier frequency according to the OLED frequency response we can achieve data rates up to $\sim 640 \mathrm{~kb} / \mathrm{s}$ at a $7 \%$ FEC limit for $f_{c}=200 \mathrm{kHz}$. This is a substantial improvement of $\sim 190 \%$ and $\sim 173 \%$ fo $f_{c}=100 \mathrm{kHz}$ and $300 \mathrm{kHz}$, respectively, due to the high attenuation below the cut-on and the cut-off frequencies. We also showed that OVLC is able to support data transmission of $160 \mathrm{~kb} / \mathrm{s}$ within a $7 \%$ FEC limit up to the tilt angle of $48^{\circ}$.

In our future work, we will focus on the optimized design of the driving circuit for OLED to reduce the attenuation at low frequencies and to achieve higher modulation index.

$$
\text { ACKNOWLEDGMENT }
$$

This work was supported by CTU grant SGS14/190/OHK3/3T/13.

\section{REFERENCES}

[1] G Haigh, P.A.; Ghassemlooy, Z.; Rajbhandari, S.; Papakonstantinou, I., "Visible light communications using organic light emitting diodes," in Communications Magazine, IEEE, vol.51, no.8, pp.148-154, August 2013

[2] J Hoa Le Minh; O'Brien, D.; Faulkner, G.; Lubin Zeng; Kyungwoo Lee; Daekwang Jung; Yunje Oh; Eun Tae Won, "100-Mb/s NRZ Visible Light Communications Using a Postequalized White LED," in Photonic
Technology Letters, IEEE, vol.21, no.15, pp.1063-1065, Aug.1, 2009

[3] Z. Ghassemlooy, P. A. Haigh, F. Arca, S. F. Tedde, O. Hayden, I. Papakonstantinou, et al., "Visible light communications: 3.75 Mbits data rate with a $160 \mathrm{kHz}$ bandwidth organic photodetector and artificial neural network equalization [Invited]," Photonics Research, vol. 1, pp. $65-68,2013$

[4] S. T. Le, T. Kanesan, F. Bausi, P. A. Haigh, S. Rajbhandari, Z. Ghassemlooy, et al., "10 Mb/s visible light transmission system using a polymer light-emitting diode with orthogonal frequency division

[5] P. A. Haigh, Z. Ghassemlooy, L.-M. Hoa, S. Rajbhandari, F. Arca, S. F. Tedde, et al., "Exploiting Equalization Techniques for Improving Data Rates in Organic Optoelectronic Devices for Visible Light Communications," Lightwave Technology, Journal of, vol. 30, pp. 3081-

[6] A. Buckley, Organic light-emitting diodes (OLEDs): materials, devices and applications: Woodhead Publishing series in electronic and optical materials, 2013.

[7] S. Reineke, F. Lindner, G. Schwartz, N. Seidler, K. Walzer, B. Lussem, et al., "White organic light-emitting diodes with fluorescent tube efficiency," Nature, vol. 459, pp. 234-238, 2009

[8] Jungnickel, V.; Uysal, M.; Serafimovski, N.; Baykas, T.; O'Brien, D.; Ciaramella, E.; Ghassemlooy, Z.; Green, R.; Haas, H.; Haigh, P.A.; Jimenez, V.P.Gil; Miramirkhani, F.; Wolf, M.; Zvanovec, S., "A European view on the next generation optical wireless communication standard," inStandards for Communications and Networking (CSCN), 2015 IEEE Conference on, vol., no., pp.106-111, 28-30 Oct. 2015

[9] IEEE, "IEEE Standard for Local and Metropolitan Area Networks--Part Light," ed: IEEE, 2012

[10] P. A. Haigh, F. Bausi, Z. Ghassemlooy, I. Papakonstantinou, H. Le Minh, C. Fléchon, et al., "Visible light communications: real time $10 \mathrm{Mb} / \mathrm{s}$ link with a low bandwidth polymer light-emitting diode," Optics Express, vol. 22, pp. 2830-2838, 2014

[11] P. A. Haigh, F. Bausi, T. Kanesan, S. T. Le, S. Rajbhandari, Z. Ghassemlooy, et al., "A 20-Mb/s VLC Link With a Polymer LED and a Multilayer Perceptron Equali
vol. 26, pp. 1975-1978, 2014

[12] Haigh, P.A.; Bausi, F.; Hoa Le Minh; Papakonstantinou, I.; Popoola, W.O.; Burton, A.; Cacialli, F., "Wavelength-Multiplexed Polymer LEDs: Towards $55 \mathrm{Mb} / \mathrm{s}$ Organic Visible Light Communications," in Selected Areas in Communications, IEEE Journal on, vol.33, no.9, pp.1819-1828, Sept. 2015

[13] A. M. Khalid, G. Cossu, R. Corsini, P. Choudhury, and E. Ciaramella, "1Gb/s Transmission Over a Phosphorescent White LED by Using Rate4, pp. 1465-1473, 2012

[14] D. Tsonev, S. Sinanovic, and H. Haas, "Novel Unipolar Orthogonal Frequency Division Multiplexing (U-OFDM) for Optical Wireless," in pp. $1-5$.

[15] P. A. Haigh, L. Son Thai, S. Zvanovec, Z. Ghassemlooy, L. Pengfei, X. Tongyang, et al., "Multi-band carrier-less amplitude and phase
The Tongyang, et al., "Multi-band carrier-less amplitude and phase
modulation for bandlimited visible light communications systems," Wireless Communications, IEEE, vol. 22, pp. 46-53,2015.

[16] Haigh, P.A.; Chvojka, P.; Zvanovec, S.; Ghassemlooy, Z.; Son Thai Le; Kanesan, T.; Giacoumidis, E.; Doran, N.J.; Papakonstantinou, I.; Darwazeh, l., "Experimental verification of visible light communications

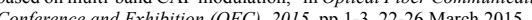

17] Fangcong Wang, Su Liu, Chunlin Zhang, The dielectric constant of materials effect the property of the OLED, Microelectronics Journal, materials effect the property of the OLED, Micro
Volume 38, Issue 2, February 2007, Pages 259-261

[18] Shafik, R.A.; Rahman, S.; Islam, R., "On the Extended Relationships Among EVM, BER and SNR as Performance Metrics," in Electrical and Computer Engineering, 2006. ICECE '06. International Conference on ,

[19] M. I. Olmedo et al., "Multiband Carrierless Amplitude Phase Modulation for High Capacity Optical Data Links," in Journal of Lightwave Technology, vol. 32, no. 4, pp. 798-804, Feb.15, 2014.

[20] Burton, A.; Le Minh, H.; Ghassemlooy, Z.; Rajbhandari, S.; Haigh, P.A., "Smart receiver for visible light communications: Design and analysis," in Communication Systems, Networks \& Digital Signal Processing 
(CSNDSP), 2012 8th International Symposium on, pp.1-5, 18-20 July [21] Chvojka, P.; Zvanovec, S.; Haigh, P.A.; Ghassemlooy, Z., "Channel Chas
Environment," in Lightwave Technology, Journal of , vol.33, no.9, pp.1719-1725, May1, 12015 